\title{
An LBP-Based Active Contour Algorithm for Unsupervised Texture Segmentation
}

\author{
M.A. Savelonas, D.K. Iakovidis, D.E. Maroulis \\ Dept. of Informatics and Telecommunication, University of Athens, \\ rtsimage@di.uoa.gr
}

\begin{abstract}
This paper presents a novel algorithm for unsupervised texture segmentation. The proposed algorithm incorporates the Local Binary Pattern operator under a segmentation framework based on the Active Contour Without Edges model. The experiments performed, show that it can be used for fast segmentation of two-textured images, outperforming recent texture segmentation algorithms, with a segmentation quality that reaches $99 \%$ on average.
\end{abstract}

\section{Introduction}

Segmentation of texture images is a major field of research in computer vision. An effective texture segmentation algorithm is of importance in diverse areas such as medical image analysis, image retrieval and remote sensing. Various structural, statistical and spectral texture segmentation approaches have been proposed [1],[2]. In particular, unsupervised texture segmentation has been the subject of intensive research in many recent studies [3]-[5].

Early segmentation approaches utilized boundarybased local filtering techniques such as edge detection operators, which require additional edge-linking operations in order to establish the connectivity of edge segments. This problem has been resolved by employing active contour models, which directly result in continuous curves. These models involve the deformation of initial contours towards the boundaries of the image regions to be segmented. A recent active contour model, named Active Contour Without Edges (ACWE) [6] has been gaining increasing interest due to its advantages: 1) it is region-based, enabling the delineation of regions defined by smooth intensity changes, 2) its level set formulation provides adaptability to topological changes, and 3) it does not require any significant initialization constraint [6]. However, in the ACWE model the contour evolution depends on the intensities rather than on the textural content of the image to be segmented.

State of the art research in active contour models has concentrated on the use of texture as a guiding force to contour evolution. Most of the previous active contour approaches for texture segmentation involved Gabor and wavelet approaches to contour evolution [5], [7], [8].

The LBP operator [9], offers an alternative approach for texture representation. Unlike the Gabor features, which are calculated from the weighted mean of pixel values over a small neighborhood, LBP considers each pixel in the neighborhood separately, providing even more fine-grained information. In addition, the LBP texture features are invariant to any monotonic change in gray level intensities, resulting in a more robust representation of textures under varying illumination conditions. Comparative studies have demonstrated that the use of LBP features may result in higher classification accuracy than the Gabor and wavelet features, with a smaller computational overhead [9]-[11].

In this paper we propose a novel algorithm for unsupervised texture segmentation that incorporates an LBP-based representation of textures under an ACWE segmentation framework.

The rest of this paper is organized in five sections. Sections 2 and 3 provide an outline of the ACWE model and the LBP features respectively. Section 4 describes the proposed algorithm. The results from its application on two-textured images are apposed in Section 5. Finally, in Section 6 the conclusions of this study are summarized.

\section{Active contour without edges}

Active Contour Without Edges as posed in [6] has the form of a minimization problem: if we consider $\Omega$ 
as a bounded open subset of $R^{2}$, with $\partial \Omega$ the boundary, we seek for $\inf F\left(c^{+}, c^{-}, C\right)$ :

$$
\begin{aligned}
F\left(c^{+}, c^{-}, C\right)=\mu \cdot \text { length }(C) & +\lambda^{+} \int_{\operatorname{in}(C)}\left|u_{0}(x, y)-c^{+}\right|^{2} \\
& +\lambda^{-} \int_{\operatorname{out}(C)}^{\mid}\left|u_{0}(x, y)-c^{-}\right|^{2}
\end{aligned}
$$

where $u_{0}: \Omega \rightarrow R$ is the given image, $C(s):[0,1] \rightarrow R^{2}$ a piecewise parameterized curve, $c^{+}$and $c^{-}$are unknown constants representing the average value of $u_{0}$ inside and outside the curve and parameters $\mu>0$ and $\lambda^{+}, \lambda^{-}>0$ are weights for the regularizing term and the fitting terms, respectively.

In the level set method [12], $C \subset \Omega$ is represented by the zero level set of a Lipschitz function $\phi: \Omega \rightarrow R$, such that

$$
\begin{aligned}
& C=\partial \omega=\{(x, y) \in \Omega: \phi(x, y)=0\}, \\
& \operatorname{in}(C)=\{(x, y) \in \Omega: \phi(x, y)>0\}, \\
& \operatorname{out}(C)=\{(x, y) \in \phi(x, y)<0\}
\end{aligned}
$$

Using the Heaviside function $H$ and the onedimensional Dirac measure $\delta$, defined respectively by

$$
\delta(z)=\frac{d}{d z} H(z), H(z)= \begin{cases}1, & \text { if } z \geq 0 \\ 0, & \text { if } z<0\end{cases}
$$

the constants $c^{+}$and $c^{-}$can be expressed as:

$$
\begin{aligned}
c^{+}(\phi) & =\frac{\int_{\Omega} u_{0}(x, y) H(\phi(x, y)) d x d y}{\int_{\Omega} H(\phi(x, y)) d x d y} \\
c^{-}(\phi) & =\frac{\int_{\Omega} u_{0}(x, y)(1-H(\phi(x, y))) d x d y}{\int_{\Omega}(1-H(\phi(x, y))) d x d y}
\end{aligned}
$$

Keeping $c^{+}$and $c^{-}$fixed, and minimizing $F$ with respect to $\phi$, we deduce the associated EulerLangrange equation for $\phi$. Parameterizing the descent direction by an artificial time $t \geq 0$, the equation in $\phi(t, x, y)$ (with $\phi(0, x, y)=\phi_{0}(x, y)$ defining the initial contour) is

$$
\begin{gathered}
\frac{\partial \phi}{\partial t}=\delta(\phi)\left[\mu \cdot \operatorname{div}\left(\frac{\nabla \phi}{|\nabla \phi|}\right)-\lambda^{+}\left(u_{0}-c^{+}\right)^{2}+\lambda^{-}\left(u_{0}-c^{-}\right)^{2}\right]=0 \\
\phi(0, x, y)=\phi_{0}(x, y) \\
t \in(0, \infty),(x, y) \in \Omega
\end{gathered}
$$

\section{Local binary pattern}

The LBP operator [9] utilizes a binary representation of texture units localized in image neighborhoods. Considering neighborhoods of $3 \times 3$ pixels, the LBP value for such a neighborhood is estimated as follows: i) the original $3 \times 3$ neighborhood (Fig.1a) is thresholded to two levels ( 0 and 1$)$ using the value of the center pixel (Fig.1b), ii) the values of the pixels in the thresholded neighborhood are multiplied by the binomial weights (Fig.1c) assigned to the corresponding pixels, iii) the values of the eight pixels (Fig.1d) are summed to obtain a single value for the corresponding pattern. Thus, a total of $2^{8}=256$ texture units are possible. The histograms of the LBP values of an image comprise discriminative texture signatures.
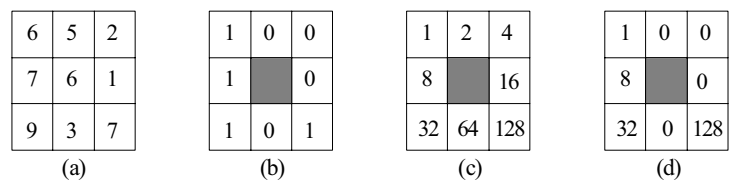

Figure 1. An example LBP value estimation: $\mathrm{LBP}=1+8+32+128=169[9]$.

\section{LBP-based active contour algorithm for texture segmentation}

The textural content of an image can be encoded by the LBP values assigned to each of its pixels. The derived LBP histogram bins are sorted according to the frequency of their occurrence in the image and the $b$ most frequent histogram bins are selected. This is in agreement with [13], according to which a subset of LBP histogram bins may perform even better than the complete histogram, for texture discrimination.

The $b$ selected histogram bins are used to generate $b$ binary images according to a simple rule: if the LBP value of a pixel located at the position $(x, y)$ of the original image belongs to the $k$-th histogram bin, then the pixel located at the position $(x, y)$ in the $k$-th generated binary image is labeled white, indicating the presence of a local texture pattern, otherwise it is labeled black. The $b$ generated binary images are combined in all possible ways by the logical OR operator (i.e. the occurrence of a white pixel in at least one of the images combined, results in a white pixel). Each of the resulting "cumulative" binary images contains partial LBP histogram information.

In the sequel, the "cumulative" binary images are segmented in properly sized blocks and the number $P_{W}(i, j)$ of the white pixels contained in each block $j$ of "cumulative" image $i$ is counted. We define the contrast index $\xi(i)$ of an image $i$ as the difference between the maximum and the minimum $P_{W}(i, j)$ :

$$
\xi(i)=\max _{j}\left(P_{W}(i, j)\right)-\min _{j}\left(P_{W}(i, j)\right)
$$


The "cumulative" binary image $i_{m}$ with the maximum $\xi$ is selected for further processing:

$$
\left.i_{m}=\underset{i}{\arg (\max }(\xi(i))\right)
$$

This image has the maximum inter-block difference in white pixel densities. Since the white pixels represent local texture patterns, the regions of different textures in $i_{m}$ are expected to be more distinguishable. At this point, it is assumed that the variance of white pixel densities within a region of the same texture is a small fraction of the variance of white pixel densities between regions of different texture.

As a next step, a Gaussian smoothing operator is applied to $i_{m}$, resulting in a smoothed image $i_{G}$. This operation reduces the local variances in the spatial frequency of the texture patterns and results in nearly homogeneous image regions that could more easily be segmented by the ACWE model. Such smoothing operations have proved to enhance texture discrimination as the notion of texture is undefined at the single pixel level although it is always associated with some set of pixels [14].

In the final step of the algorithm, the ACWE model is applied to $i_{G}$. The region-based formulation of this active contour model enables the segmentation of an image into discrete regions, even if the regions are not explicitly defined by high intensity gradients. It is worth noting that the ACWE algorithm reaches a global minimizer [6] and therefore the segmentation achieved is independent from the initial contour.

The steps of the proposed algorithm can be summarized as follows:

1. Calculate the LBP histogram of an image

2. For each of the $b$ most frequent LBP histogram bins generate a binary image do

For each pixel of the binary image do

If the LBP pattern of the corresponding LBP histogram bin is present, then pixel is white else pixel is black

3. Build "cumulative" binary images $i$ by applying the logical OR operator on all combinations of the $b$ binary images.

4. Find $i_{m}$ using (8)

5. Filter $i_{m}$ with a Gaussian operator to produce $i_{G}$

6. Segment $i_{G}$ using ACWE.

\section{Results}

In our experiments, we applied the proposed algorithm to segment 12 two-texture images, composed of natural textures. The same composite images have been used in [15],[16].
The proposed algorithm was implemented in Microsoft Visual C++ and executed on a $3.2 \mathrm{GHz}$ Intel Pentium IV workstation. The ACWE constants were generally chosen as follows: $\lambda^{+}=5, \lambda^{-}=5, \mu=650$, whereas a $5 \times 5$-pixel neighborhood was considered for the calculation of the LBP values. The Gaussian smoothing parameter $\sigma$ was set to 4 and the block size was set to $16 \times 16$. Finally, for the step 2 of the algorithm, $b=5$ generated binary images were found to be sufficient for the performed segmentation tasks.

In accordance with [16], the percentage of the correctly classified pixels $q$ can be considered as a segmentation quality measure. The average segmentation quality achieved in terms of $q$ is $98.7 \pm 0.4 \%$. From another viewpoint, the segmentation quality can be measured by the overlap value $v$ [17] describing the overlap between the area $A$ delineated by the contour and the "ground truth" area $G$. The average segmentation quality achieved in terms of $v$ is $92.4 \pm 0.9 \%$. Each run of the proposed algorithm lasts approximately 4 seconds, which is less than the execution times required by other algorithms proposed in recent studies [2][8].

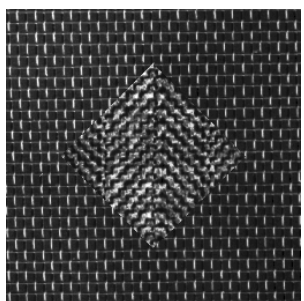

$\left(a_{1}\right)$

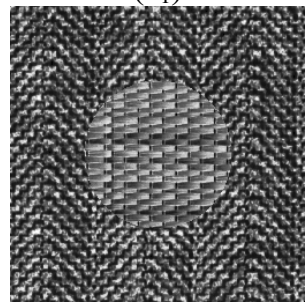

$\left(a_{2}\right)$

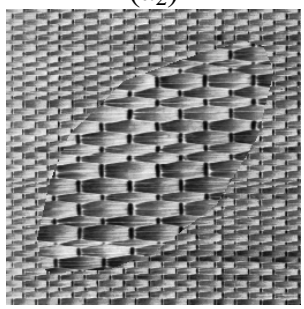

$\left(a_{3}\right)$

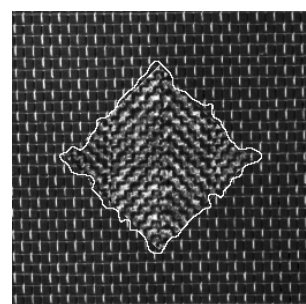

$\left(b_{1}\right)$

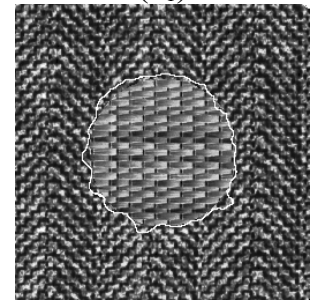

$\left(b_{2}\right)$

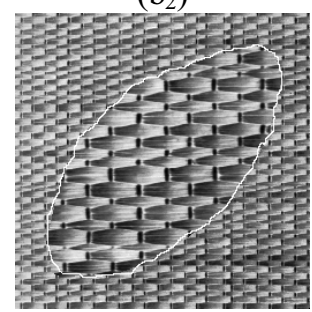

$\left(b_{3}\right)$
Figure 2. Three example images containing discrete texture regions: $\left(a_{1}-a_{3}\right)$ original two-texture images, $\left(b_{1-}\right.$ $b_{3}$ ) the final contours at convergence. 
Figure 2 illustrates the results of the application of the proposed algorithm on three examples of twotexture images. The proposed algorithm achieves a $q$ of $90.0 \%, 98.6 \%$, and $98.1 \%$ for the examples illustrated in Fig. $2 b_{1}, 2 b_{2}$, and $2 b_{3}$ respectively. The percentage in Fig. $2 b_{2}$ is higher than $98.3 \%$, which was reported as the best result on the comparative evaluation included in [16], with respect to the same image.

\section{Conclusion}

We have proposed a novel LBP-based active contour algorithm for texture segmentation. The algorithm embodies the advantages of LBP and ACWE methodologies. In our experimental study, the proposed algorithm achieved very promising segmentation results, outperforming the best results reported on the comparative evaluation included in [16], with respect to the same images. The proposed algorithm does not impose initialization constraints, and its execution time is less than the execution times required by other algorithms proposed in recent studies [2], [8].

Future perspectives of this work include: 1) further optimization of the algorithm implementation to reduce execution time, 2) extension of the proposed algorithm for the segmentation of multiple-texture images by incorporating the multi-phase ACWE [18], 3) the development of a methodology that will allow for automatic parameter tuning of the algorithm and the application of the proposed algorithm to medical images in which texture is dominant and useful in diagnosis, and 4) the utilization of alternative metrics that describe the global distribution of white pixel densities for each "cumulative" image, as an alternative to contrast index $\xi(i)$ in (7).

\section{Acknowledgement}

This work was supported by the Greek General Secretariat of Research and Technology and the European Social Fund, through the PENED 2003 program (grant no. 03-ED-662).

\section{References}

[1] S. Theodoridis, K. Koutroumbas, "Pattern Recognition", Academic Press, 1998.

[2] M. Mirmehdi, M. Petrou, "Segmentation of Color Textures", IEEE Trans. PAMI, vol. 22, no. 2, 2000, pp. $142-$ 159.

[3] X. Quing, Y. Jie, D. Siyi, "Texture Segmentation using LBP embedded Region Competition", El. Letters on Comp. Vision and Image Anal., vol. 5, no.1, 2005, pp. 41-47.
[4] M. Rousson, T. Brox, R. Deriche, "Active Unsupervised Texture Segmentation on a Diffusion Based Feature Space", Proc. IEEE CCVPR, Madison, Wisconsin, USA, 2003.

[5] C. Sagiv, N.A. Sochen, Y. Zeevi, "Integrated Active Contours for Texture Segmentation", IEEE Trans. Image Processing, vol. 1, no. 1, 2004, pp. 1-19.

[6] T.F. Chan, L.A. Vese, "Active Contours Without Edges", IEEE Trans. Image Processing, vol. 7, Feb. 2001, pp. 266-277.

[7] N. Paragios, R. Deriche, "Geodesic Active Contours for Supervised Texture Segmentation”, Proc. IEEE International Conference on Computer Vision and Pattern Recognition, 1999, pp. 2422-2427.

[8] J.F. Aujol, G. Aubert, L. Blanc-Feraud, "Wavelet-Based Level Set Evolution for Classification of Textured Images", IEEE Trans. on Image Processing, vol. 12, no. 12, 2003, pp. 1634-1641.

[9] T. Ojala, M. Pietikainen, D. Harwood, "A Comparative Study of Texture Measures with Classification based on Feature Distributions", Pattern Recognition, vol. 29, 1996, pp. 51-59.

[10] P. Paclic, R. Duin, G.V. Kempen, R. Kohlus, "Supervised Segmentation of Textures in Backscatter Images", Proc. IEEE International Conference on Pattern Recognition, vol. 2, 2002, pp. 490-493.

[11] D.K. Iakovidis, D.E. Maroulis, S.A. Karkanis, "A Comparative Study of Color-Texture Image Features," in Proc. IEEE Int. Workshop on Systems, Signal and Image Processing (IWSSIP), Halkida, Greece, 2005, pp. 205-209.

[12] S. Osher, J. Sethian, "Fronts Propagating with Curvature- Dependent Speed: Algorithms Based on the Hamilton-Jacobi Formulations", Journal Of Computational Physics, vol. 79, 1988, pp. 12-49.

[13] T. Maenpaa, The Local Binary Pattern Approach to Texture Analysis- Extensions and Applications, Oulou University Press, Finland, 2003.

[14] M. Unser, M. Eden, "Nonlinear Operators for Improving Texture Segmentation Based on Features Extracted by Spatial Filtering", IEEE Trans. On Systems, Man and Cybernetics, vol. 20, no. 4, 1990, pp. 804-815.

[15] T. Randen, and J. H. Husøy, "Filtering for Texture Classification: A Comparative Study," IEEE Transactions on Pattern Analysis and Machine Intelligence, vol. 21, no. 4, pp. 291-310, 1999.

[16] M. Acharyya, M.K. Kundu, “An Adaptive Approach to Unsupervised Texture Segmentation Using M-Band Wavelet Transform", Signal Processing, vol. 81, 2001, pp. 13371356.

[17] X. Hao, C. Bruce, C. Pislaru, and J.F. Greenleaf, “A Novel Region Growing Method for segmenting ultrasound images", IEEE International Ultrasonics Symposium, vol. 2, 2000, pp. 1717-1720.

[18] L.A. Vese, T.F. Chan, "A Multiphase Level Set Framework for Image Segmentation Using the Mumford and Shah Model", It. J. Comp. Vision, vol. 50, no. 3, 2002, pp. 271-293. 